\title{
Sandbox regulatorio como fuente de derecho en caso de la disrupción nanotecnológica brasileña
}

\section{The regulatory sandbox as a source of law in the nanotech disruption case in Brazil}

\author{
Daniela Pellin,*,* Wilson Engelmann**
}

\begin{abstract}
This article aims to consolidate the regulatory sandbox as a source of normative production to address the legal regulation of the development and application of nanotechnology. The paradox that exists between nanotechnology and risk, both inherent to development, is presented as a problem for society as it must deal with limits. For this, a legal regulation is expected. But, what legal regulation is being considered? Certainly, that produced by social intervention as a factor for validating and managing the limits of this paradox. In this sense, following another Brazilian initiative, The regulatory sandbox is presented as a possibility of legal regulation of the development and application of nanotechnology. To achieve this objective, it uses the project of the Brazilian securities commission that, together with the Inter-American Development Bank, implemented the so called sandbox normative process in order to legally regulate technology-based financial companies at an early stage. In addition, meeting the needs of risk control and favoring the development of society. The methodology used is hypothetical-inductive which qualitative approach and with a systemic-constructivist approach. As a research technique, it uses data collection, document and literature review. The results suggest that in the case of nanotechnology development and given the impossibility for the constituent legislator to know such an object, the regulatory sandbox can enable a dynamic regulation and democratic norms where all actors participate in the construction of the regulatory process, therefore impacting decision-making about innovation.
\end{abstract}

KEYWORDS: nanotechnology, regulatory sandbox, regulatory impact analysis, source of law.

RESUMEN: Consolidar el sandbox regulatorio como fuente de producción normativa para abordar la regulación legal del desarrollo y aplicación de la nanotecnología es el objetivo de este artículo. Dada la paradoja existente entre la nanotecnología y el riesgo, ambos inherentes al desarrollo, la cual se presenta como un problema para la sociedad al tener que lidiar con límites, sería deseable conseguir una regulación legal. Pero, ¿qué regulación legal se está considerando? Ciertamente, a la producida por la intervención social como factor de validación y gestión de los límites de esta paradoja. En este sentido, y retomando otra iniciativa brasileña, el sandbox regulatorio se muestra como una posibilidad de regulación legal del desarrollo y aplicación de la nanotecnología. Para lograr este objetivo, nos basaremos en el proyecto de la Comisión de Valo-

Recibido: 25 de febrero, 2021. Aceptado: 9 de agosto, 2021. Publicado: 17 de septiembre, 2021.

* Universidade do Vale do Rio dos Sinos. Programa de Pós-Graduação em Direito do Mestrado Profissional em Direito da Empresa e dos Negócios.

** Universidade do Vale do Rio dos Sinos. Programa de Pós-Graduação em Direito Acadêmico (Mestrado e Doutorado) e Coordenador Executivo do Mestrado Profissional em Direito da Empresa e dos Negócios.

•Autora de correspondencia: dpellin@unisinos.br 
res de Brasil, la cual, junto con el Banco Interamericano de Desarrollo, implementó el proceso normativo denominado sandbox, para regular legalmente las empresas financieras de base tecnológica en una etapa temprana, además de atender las necesidades de control de riesgos y favorecer el desarrollo de la sociedad. La metodología utilizada es hipotético-inductiva, de naturaleza cualitativa y de enfoque sistémico-constructivista. Como técnica de investigación, se utiliza la recolección de datos, documentos, y el análisis de literatura. Los resultados indican que en el caso del desarrollo nanotecnológico y ante la imposibilidad de que el legislador constituyente conozca tal objeto, el sandbox regulatorio puede dar paso a normas dinámicas y democráticas donde todos los actores participan en la construcción del proceso regulatorio, impactando, por tanto, la toma de decisiones sobre la innovación.

PALABRAS CLAVE: nanotecnología, sandbox regulatorio, análisis del impacto normativo, fuente del derecho.

\section{Introducción}

El complejo sistema que impregna el desarrollo nanotecnológico es de difícil regulación en tanto parte del conjunto de nuevas tecnologías que componen el complejo de la denominada Revolución 4.0 la cual habilita el auge de la industria 4.0 (Schwab, 2016 y 2018). Las nanotecnologías se desarrollan en diversas áreas científicas y se tratan como insumos productivos para la industria 4.0, cuyos resultados son ahorros de costos, ganancias en la eficiencia productiva y mejores resultados para el consumidor. El problema radica en el control legal y regulatorio sobre el riesgo y el desarrollo nanotecnológico junto con las numerosas recomendaciones técnicas producidas a nivel mundial, no obligatorias, pero indicativas. El objetivo general es demostrar que la lógica sistémica y el lenguaje comunicativo de la corregulación del carácter experimental propuesto por el sandbox deben ser aplicados y tratados como fuente del derecho para el tratamiento legal de las nanotecnologías. La hipótesis es que de este modo será factible regular el sistema y equilibrar las fuerzas del juego económico: desarrollo, riesgo, consumo sostenible y democracia. Esto es posible porque, desde que se promulgó en Brasil la Ley 13.874, de 20/9/2019, cualquier iniciativa regulatoria debe ser sometida al análisis de impacto normativo para frenar el abuso regulatorio o su imposibilidad, cuyo propósito es detener el incumplimiento, contribuir con la garantía de los derechos fundamentales y con los objetivos de implementar políticas públicas. Por tanto, la democratización del proceso normativo, al favorecer la participación de todos los interesados, puede implicar la construcción de normas y el cumplimiento voluntario de las reglas, actuando como fuente del derecho para insertarse en el ordenamiento jurídico.

\section{El escenario del diseño metodológico de la regulación de la nanotecnología}

En la academia jurídica, se ha discutido ampliamente la posibilidad o imposibilidad del derecho actual para atender las necesidades de la sociedad de la información (Brasil, 2000) y las tecnologías disruptivas de la industria 4.0. 
Las nuevas tecnologías son ciertamente propiedad del sistema económico, captadas por la perspectiva de desarrollo y crecimiento exponencial, y mismas que colocan a Brasil en la condición de proveedor de bienestar y competidor internacional (Pellin, 2019).

Por otra parte, la nanotecnología es parte de una agresiva ruptura histórica; se la trata como insumo productivo, desarrollada y aplicada en diversos sectores como el farmacéutico, el químico, físico, médico, o en robótica, por mencionar algunos. Considerando el ínfimo tamaño de un nanómetro $(\mathrm{nm})$, invisible a simple vista, y con una dimensión de $1 \mathrm{~nm}=10^{-9} \mathrm{~m}$, es posible, utilizando nanotecnología, alterar las propiedades de los materiales desde los átomos y las moléculas y, en consecuencia, modificar las características y propiedades de los productos que los utilizan. Por esta razón, los resultados pueden ser increíbles, tanto en nuevos materiales, como en términos de nuevas eficiencias, nuevas economías, y nuevos modelos o formas de consumo. Pese a ello, es notorio que no se dedique la misma energía a la evaluación e investigación de los riesgos de la posible interacción de estos materiales nanoestructurados cuando entran en contacto con la salud humana y la preservación del medio ambiente (Pellin, 2019).

En la prisa de Brasil por superar el retraso tecnológico reflejado en el desarrollo (Beck, 2011), la toma de decisiones sobre qué desarrollar e innovar no adopta como premisa la evaluación de riesgos, indispensable para la seguridad del desarrollo sostenible.

Tomando en cuenta el diagnóstico anterior, se busca, a través del derecho, una solución política, económica, técnica y científica para equilibrar las fuerzas de la economía. Haciendo un seguimiento de todas las investigaciones realizadas hasta el momento, es posible darse cuenta de que existe un vacío jurídico que es llenado por nuevos patrones de comunicación y lenguaje, y que se refieren al deber de promover el desarrollo económico y sostenible, así como a la aplicación del principio de precaución en la toma de decisiones económicas (Pellin y Engelmann, 2018). Entonces, ¿por qué esta perspectiva no es atendida en Brasil? La respuesta es simple: se trata de un país de modernidad tardía (Beck, 2011) y bajo un patrón sistémico cultural (Pellin y Engelmann, 2018) que impide el avance tecnológico seguro y deseable.

Por ello, la investigación es particularmente necesaria en este ámbito, con miras a atender el escenario disruptivo como un concepto que significa interrumpir, reiniciar un proceso, e impulsar una propuesta nueva, diferente, innovadora, inventiva, que las tecnologías traen consigo en esta era de la Revolución industrial 4.0 (Schwab, 2016). Se exige también del derecho que sea disruptivo, que provoque una ruptura en el precepto histórico de su construcción ontológica de significado, permitiendo la innovación en los procesos creativos de nuevas fuentes del propio derecho.

Como impacto de este escenario de innovación tecnológica, la Comisión de Valores de Brasil ideó la aplicación de la figura del sandbox en el caso de las FinTechs (tecnologías financieras: las denominadas empresas financieras de 
base tecnológica que operan en territorio virtual) como medio para enfrentar el riesgo, el desarrollo y la regulación legal eficiente, capaz de conformar comportamientos de manera voluntaria y adherida por todos los interesados en el sector.

El sandbox surgió en Estados Unidos en 2012 de la mano de la Oficina de Protección Financiera del Consumidor. Después de tres años, el organismo responsable del mercado financiero británico, la Financial Conduct Authority, se ha sumado al modelo.

El término sandbox viene de la informática. Se trata de establecer un entorno virtual para la experimentación de nuevos programas en sistemas operativos ya funcionales. En la práctica, los límites de su alcance están prestablecidos para que, si hay fallos en el programa probado, el sistema no se vea comprometido (Feigelson et al., 2019). Se trata, por tanto, de una metodología de experimentación que, en este caso, se nutre de una regulación y un control supervisados, pero flexibles en la medida en que uno puede soportar el régimen jurídico que envuelve su actividad. Hoy en día, hay más de veinte países adheridos al proceso de regulación como política pública de incentivos y control (Jenik y Lauer, 2017).

Se ha comprobado la posibilidad de aplicar el mismo acercamiento para tratar los asuntos legales de la regulación de las nanotecnologías. Adicionalmente, en este proceso regulatorio, todos los actores deben estar involucrados y capturados por la autorregulación común. Es decir, la corregulación que involucra al Estado democrático y a los múltiples actores e intereses involucrados. Como resultado, se eleva el nivel sistémico deseado, comprometido y responsable, y se construye una respuesta efectiva que permita al sistema jurídico, sus operadores y a los tribunales responder adecuadamente al avance de la industria 4.0.

\section{El contexto de la nanotecnología y algunas implicaciones del desarrollo}

Las nanotecnologías son lo que se ha llamado nuevas tecnologías disruptivas, entendidas por tanto como aquellas que cambian los patrones culturales de una sociedad, en términos de economía, entretenimiento, relaciones, comunicación, consumo, política, gobierno, por mencionar algunos. El cambio está en todas partes, por lo cual, según Schwab (2016), se inaugura una nueva era - la Cuarta Revolución Industrial: la Revolución 4.0-. Se trata de cambios no vistos con anterioridad y dándose a una marcada velocidad, amplitud y profundidad. Son cambios de impacto sistémico que fusionan inteligentemente las nuevas tecnologías, contexto en el cual se denota la presencia de interacciones entre los dominios físico, digital y biológico.

En el caso de las nanotecnologías desarrolladas para impactar en las propiedades de los materiales, haciéndolos más resistentes, posibles de reciclar y adaptables con aplicaciones inteligentes de autorreparación y autolimpieza; 
metales con memoria para volver a su forma original; cerámicas y cristales que transforman la presión en energía, entre otros, conjuntan algunos de los componentes de la canasta de inversiones, con perspectivas de desempeñar el papel fundamental en la "mitigación de los riesgos globales que enfrentamos" (Schwab, 2016: 26).

En este escenario, la prisa por la investigación y la competencia global conjugan el locus del desarrollo de las nanotecnologías junto con los riesgos para los sistemas naturales y sociales ante las incógnitas o las incertidumbres científicas sobre las interacciones de los nanomateriales con los humanos y el medio ambiente, comprendidas en toda su complejidad (Pellin, 2019). Muchas implicaciones están involucradas en esta complejidad, especialmente en lo que respecta a las condiciones sensibles de los países en desarrollo como Brasil, aún distante del ideal de acuerdo con los estándares de la globalización económica (Brasil, 2000). Se constata, cuando se trata de las mejoras en el perfil empresarial, cultural y educativo del país, que el nivel de Brasil está considerablemente por debajo de los patrones razonables (Cai, 2013), y no tiene las condiciones para apropiarse del conocimiento y la innovación por encima de los patrones deseables en la Constitución Federal de 1988, apoyados en el escenario competitivo global y la política nacional de innovación. Además, los innumerables requerimientos a ser enfrentados y contraídos — según Beck (2011), por la modernidad posterior - cuestionan el avance de la nanotecnología sin regulación específica, al proyectar un escenario sin control y de mayor riesgo.

Después de la edición del Libro verde (Brasil, 2000), en el que el país asumió el compromiso global de ajustarse a los patrones de la sociedad de la información; en 2002 se publicó la edición del Libro blanco (Brasil, 2002), donde se traza un programa nacional de desarrollo del país de 2001 a 2012 en materia de ciencia, tecnología e innovación, demarcando como objetivo la reducción de las desigualdades regionales como distintivo de justicia y bienestar social. Además, en esta perspectiva de desarrollo, se incluyeron las empresas socias del Estado, tanto las nacionales, de cualquier tamaño, como las extranjeras en territorio nacional.

Es del Libro blanco, consecuencia del Libro verde, del que se extrae el concepto de innovación brasileña, entendido como "un fenómeno complejo y multidimensional, que presupone la presencia y articulación de un elevado número de agentes e instituciones de variada naturaleza con lógicas y procedimientos distintos; metas de corto y largo plazos; potencialidades específicas, restricciones y motivaciones diversas" (Brasil, 2002: 26). Sin embargo, con el Libro azul (Brasil, 2010) se ha reforzado este concepto considerando la propuesta del país de adoptar como objetivo estratégico el desarrollo científico y tecnológico innovador, basado en una política de reducción de las desigualdades regionales y sociales, exploración sustentable de la riqueza del territorio nacional y fortalecimiento de la industria. En consecuencia, añadiría valor a la producción y exportación a través de la innovación y el refuerzo de la importancia internacional de la ciencia y la tecnología. 
La agenda principal para la concretización del Libro azul es propiciar la innovación para el consumo masivo:

La economía brasileña se encuentra en una fase especial de su trayectoria histórica. Hay evidencias inequívocas de que en los últimos años se ha iniciado un proceso que probablemente se afirme como un nuevo ciclo de desarrollo a largo plazo: el crecimiento con redistribución de la renta a través de la dinámica de la producción y el consumo de masas. Se trata de un viejo sueño de la sociedad brasileña, que se presenta en el momento actual de la vida nacional como una tendencia prometedora y absoluta. (Brasil, 2010: 29).

Los datos confirman que esta política pública gubernamental ha funcionado y acelerado el proceso de acceso a los bienes y servicios de consumo masivo. Algunos sectores, como el sector industrial de los cosméticos, ganan visibilidad, pues con la innovación nanotecnológica aplicada a los procesos y productos de higiene personal, cosmética y perfumería, se destacó a nivel nacional e internacional.

La Asociación Brasileña de la Industria de la Perfumería, Cosmética e Higiene Personal presentó en el documento institucional Panorama Sectorial de 2019 (ABIHPEC, 2019) datos relacionados con el desempeño sectorial. Según se informó, se destina un millón de reales a inversiones que marcan huella en la economía brasileña en el plazo de un año, con impactos socioeconómicos considerables en comparación con otros sectores industriales como la agroindustria e industrias en general (ABIHPEC, 2019: 42), (tabla 1).

Brasil es el cuarto mercado mundial de consumo de productos de higiene personal, perfumería y cosmética, con una cuota de mercado global del $6.2 \%$ y un total de 30 mil millones de dólares en ventas al consumidor en 2018, según la información recogida por ABIHPEC de Euromonitor. El país está solo detrás de Estados Unidos (EUA) con el 18.3\% (89,500 millones de dólares), China con 12.7\% (62,000 millones de dólares) y Japón con 7.7\% (37,500 millones de dólares). En América Latina, Brasil tiene el 48.6\% del mercado de la región y en cuanto al mercado mundial, América Latina tiene el 12.7\% (ABIHPEC, 2019: 43). Hace tiempo que es el sector que más invierte en investigación, desarrollo e innovación: 1.9 millones de reales en 2018; así como el sector que más in-

TABLA 1. Desempeño de la industria de la perfumería, cosmética e higiene personal (PCPH) en Brasil según datos reportados en 2019.

\begin{tabular}{|c|c|c|c|}
\hline $\begin{array}{c}\text { Impactos } \\
\text { socioeconómicos }\end{array}$ & PCPH & Agronegocios & Industria en general \\
\hline Producción & 3.85 millones de reales & 2.96 millones de reales & 3.72 millones de reales \\
\hline Ocupación & 38 puestos de trabajo & 80 puestos de trabajo & 37 puestos de trabajo \\
\hline Impuestos & $601 \mathrm{mil}$ & 171 mil & 354 mil \\
\hline Salario & $450 \mathrm{mil}$ & $340 \mathrm{mil}$ & 421 mil \\
\hline
\end{tabular}

Fuente: Elaboración propia con base en ABIHPEC (2019). 
vierte en publicidad con 9.3 millones de reales en la marca y 3.7 millones de reales en activos (ABIHPEC, 2019: 43).

Actualmente, hay 2,794 empresas registradas en la Agencia Nacional de Vigilancia Sanitaria. La mayor concentración se encuentra en el sudeste, con 1,685 , seguido por el sur, con 550, el noreste, con 309, medio oeste con 197 y el norte, con 55. Cabe precisar que en 2018, hubo un aumento del $2.8 \%$ en el número de empresas registradas en la Agencia, debido a la necesidad de mantenerse al día con la competitividad del mercado, la demanda de innovación, calidad, seguridad y efectividad de productos (ABIHPEC, 2019: 43).

Es un mercado prometedor en marcha y con perspectivas de progreso y crecimiento exponencial por lo que los riesgos no pueden ser marginados. No obstante, el sector en Brasil no enfrenta su responsabilidad extendida. Un ejemplo de ello son los dos Proyectos de Ley (Brasil, 2013) que fueron archivados en la Cámara de Diputados, a pesar de que la exposición de motivos reforzaba los riesgos desconocidos para los seres humanos (consumidor) y el medio ambiente, así como la necesidad de una información clara y precisa para el consumidor sobre productos de nanoingeniería.

En la Cámara de Diputados, el Proyecto de Ley 6741/2013, ingresado el 31 de enero de 2019, trató sobre la implementación de la Política Nacional de Nanotecnología, la producción, el destino de los rechazos y el uso y aplicación de las nanotecnologías en el país. La proposición de Ley 5133/2013, trataba sobre el etiquetado de productos hechos con nanotecnología. En el Senado Federal, el proyecto de Ley 131/2010, presentado el 1 de agosto de 2013, trató de las determinaciones a la vigilancia sanitaria del etiquetado y el seguimiento de los medicamentos, drogas, insumos y correlativos farmacéuticos, cosméticos, agentes sanitizantes y otros productos producidos con el uso de la nanotecnología, especialmente en lo que respecta a la información en las etiquetas, prospectos y materiales publicitarios. Del mismo modo, la Agencia Nacional de Vigilancia Sanitaria, al tomar la iniciativa de regular el sector en la agenda normativa 2013-2015, archivó el procedimiento por tener temas más importantes que tratar en ese momento, aunque destacó en el documento institucional, la temeridad del desarrollo nanotecnológico sin medición y control científico (Pellin, 2019).

El hecho es que en el ambiente en que ocurren las revoluciones científicas, las probabilidades de las innovaciones tecnológicas son variables, justamente porque las revoluciones traen consigo "episodios de desarrollo no acumulativo, en los que un paradigma más antiguo es sustituido total o parcialmente por uno nuevo, incompatible con el anterior"(Kuhn, 2013: 177), promoviendo un escenario de crisis en el que se restructura la política y el proceso de toma de decisiones en torno a las perspectivas del desarrollo, dígase, por ejemplo, en Brasil, un país, como se dijo, de modernidad tardía (Beck, 2011). Además, en la modernidad ello ha significado la priorización del desarrollo económico a través de la innovación tecnológica sobre la certidumbre científica para preservar un cierto nivel de soberanía nacional y competitividad global, incluso si los desajustes 
son compartidos internamente por toda la comunidad. Este proceso debe incluir el conocimiento, el consentimiento y el respaldo de la OCDE (Pellin, 2019).

Con un reflejo directo, el escenario jurídico también se ve impactado por la revolución tecnológica, sobre todo teniendo en cuenta si es disruptiva, como en el caso de Revolución 4.0 en la que se destaca la nanotecnología. Dado que esta altera radicalmente el sistema socioeconómico en su conjunto, lo hace, en consecuencia, también en el derecho, el cual ha sido alcanzado por este proceso y forzosamente modificado. A nivel de la revolución tecnológica y la explotación económica del desarrollo científico, lo que hay en el derecho refleja el mismo entorno tecnológico, porque el sistema positivo del derecho no está en condiciones de incorporar las innovaciones tecnológicas con el marco jurídico que en su día se construyó. Se trata de un periodo de ruptura con los viejos paradigmas, en todos los sentidos. Por lo tanto, la fuente directa - la ley (Art. 4, Ley de Introducción a las Normas del Derecho Brasileño-LINDB) - no puede regular el movimiento de innovación tecnológica en constante dinamismo, en tanto que los legisladores no tengan las condiciones técnicas suficientes para tal alcance.

Asumir que las fuentes indirectas del derecho como los principios generales, la analogía y la costumbre (Art. 5, LINDB) pueden albergar innovaciones de cualquier tipo y que los tribunales pueden atender las lagunas en caso de conflictos es simplemente una ilusión, pues hay que tomar en cuenta que lo nuevo que se experimenta no tiene antecedentes históricos y, en consecuencia, no tiene condiciones para proporcionar una situación análoga razonable y coherente ni responde al transito socioeconómico en la sociedad de la información. Lo anterior se debe a que existe una relación directa de equivalencia entre las alternativas que tienen los tribunales para juzgar, derivadas de las fuentes del derecho y la asimetría de información existente en la cadencia de hechos nanotecnológicos que necesariamente escaparán a este funcionamiento del sistema jurídico por este universo desconocido.

Los tribunales juzgan por el conocimiento que tienen del derecho disponible en el sistema jurídico una vez cerrado en sí mismo y dependiente del lenguaje de comunicación interna (fuentes directas e indirectas) que ha creado para servir a la sociedad (Bobbio, 2006). Ocurre que en este cierre, los nuevos acontecimientos, como el universo nanotecnológico - que no tiene conformación jurídica-, pueden carecer de respuestas judiciales o atenderlas erróneamente. De ahí la necesidad de que múltiples actores ajenos a estas fuentes tradicionales del derecho encuentren y fijen estándares normativos para la nanotecnología de modo que, al construirlos, los tribunales dispongan de alternativas legales coherentes y razonables para aplicarlos. En este sentido, Luhmann (2016) considera que:

Con la ayuda de la relación asimétrica entre la legislación y la jurisprudencia, y de los medios conceptuales derivados (como la doctrina de las fuentes del derecho), se pretende evitar la circularidad que supondría admitir que el Tribunal 'crea' el derecho, 
aunque 'lo aplique'. [...] Se da la circunstancia de que el círculo no se produciría si los tribunales, en situaciones en las que no pueden 'encontrar' el derecho, pudieran conformarse con un no-límite (no está claro). Sin embargo, esto no se les permite -y no se les permite desde el punto de vista del derecho. (Luhmann, 2016: 409-410).*

Finalmente, permitir la ciencia del derecho (Miranda, 1972), lograda a través de la interpretación y argumentación jurídica construida por los operadores del sistema jurídico interno, incluyendo los tribunales, es insatisfactorio, dada la distancia, el vacío temporal (Ost, 1999), y la brecha entre los hechos tecnológicos y la expectativa de una respuesta jurídica eficiente y eficaz. De acuerdo con las necesidades que presenta este escenario, en contraste con la falta de conocimiento técnico, la velocidad, las disrupciones, el diagnóstico y el pronóstico, hay que considerar que:

[...] el tiempo vivido no es en tiempo real: a veces puede ser más rápido; a veces puede ser más lento. Las transformaciones del mundo que hemos vivido en los últimos años, ya sea por la crisis que precipitó un sistema de poder que parecía muy sólido y que pretendía representar el futuro del planeta, o por la rapidez del progreso técnico, nos dan el doble acortamiento y aceleración de los tiempos. (Bobbio, 2004: 94).*

Los investigadores, en sintonía con la propuesta de Revolución 4.0, deben contribuir, no solo con el tratamiento de las fuentes del derecho que está ahí, asomándose a nuevas perspectivas, si es posible, sino también insertando otras fuentes normativas, dentro de este sistema global, en la construcción de otro derecho. También deberían cuestionar el sistema interno, que necesita mantener las bases de soberanía bien definidas.

En esta nueva era del derecho y en esta era de nuevos derechos, se dice que "el derecho es una figura deóntica y, por tanto, es un término de lenguaje normativo, es decir, un lenguaje en el que se habla de normas sobre normas" (Bobbio, 2004: 94). Esto significa que no se trata de una emanación del poder estatal que se debilita (Bobbio, 2004: 24), ni derechos que corresponden directamente a obligaciones legislativas, sino, más bien, “(...) de la existencia de un poder normativo, en el que por 'existencia' se entiende tanto el mero factor externo de un derecho histórico o existente como el reconocimiento de un conjunto de normas como guía para la propia acción" (Bobbio, 2004: 94). Esto se refleja en la ampliación del espectro de legitimidad a la construcción normativa, es decir, de los múltiples actores sociales que se apropian de la Declaración Universal de los Derechos Humanos, y que comienzan a regular conductas colectivas como fuentes de derecho que, a su vez, deben insertarse en el sistema jurídico con reconocida duración, vigencia y eficacia, capaz de generar deberes como expresión del propio derecho.

* Traducción de los autores. 


\section{El sandbox regulatorio y la viabilidad de la propuesta sobre el caso de la nanotecnología}

En el universo del sistema tecnocientífico, locus de las nanotecnologías, existe una propuesta pluralista de la que se derivan valores, principios y reglas que pretenden establecer la estructura jurídica a partir de la cual se erige la Industria 4.0 y, con ella, la organización de los estándares de lenguaje y comunicación de este sistema. Las operaciones de este sistema deben estar respaldadas por las estructuras normativas, garantizando la seguridad del propio sistema, así como de sus usuarios y de los sistemas ambientales y sociales en toda su plenitud sistémica.

Este régimen normativo plural (Pellin, 2019) que abarca el universo de desarrollo y aplicación de la nanotecnología contiene, por un lado, principios europeos (EU, 2009) y mundiales (OCDE, 2015) y, por otro, normas técnicas emanadas de la Organización Internacional de Normalización (ISO, 2005). También cuenta con normas constitucionales internas, Derechos Humanos (Ruggiu, 2011) y los Objetivos de Desarrollo Sostenible (ODS), previstos en la Agenda 2030 (Pellin y Engelmann, 2019), que deben respetarse y cumplirse en cada etapa del desarrollo nanotecnológico y en cada fase del ciclo de vida de los nanomateriales y nanoproductos.

Se trata de un régimen jurídico y técnico que no emana del poder estatal como agente político de coerción, sino de estructuras de valores y principios que surgen colectivamente de múltiples actores que pretenden conformar -y de hecho confirmar - comportamientos colectivos a partir de la comunicación generalizada y sectorial de dichas estructuras. Hay un cambio significativo en los patrones culturales, en este caso, legales y técnicos del sistema en el que se inserta la comunicación estructural. Sucede que las estructuras solo tienen valor real cuando se utilizan para la asociación de eventos comunicativos; las normas, solo en la medida en que se citan explícita o implícitamente; las expectativas, únicamente en el momento en que se expresan a través de la comunicación (Luhmann, 2016). Y, como resultado de esta operación de comunicación de valores y principios por parte de múltiples actores del sistema, surge un sistema jurídico más acorde con la realidad circundante de la Industria 4.0.

Según Jasanoff (2011), la "Ciencia Reguladora” es un dominio distinto de la producción científica, responsable de las demandas epistémicas y normativas de una manera que explica su vulnerabilidad a los desafíos tanto de la ciencia como de la política. En este universo de comunicación científica, técnica y política, Engelmann sostiene que es necesaria la construcción de una "Ciencia Reguladora" que pueda estructurar este pluralismo de intereses, capaz de orientar el desarrollo y aplicación de las nanotecnologías para abarcar todo el ciclo de vida de un producto o proceso que hace uso de la nanotecnología (Engelmann, 2018). Desde esta perspectiva y como una de las formas de producción normativa, una de las posibilidades emergentes reside en el sandbox 
insertado en el sistema regulatorio interno. La legitimidad de la propuesta ha sido confirmada en Brasil con la reforma del la LINDB (Art. 20 a 30), la edición de la Ley de Libertad Económica No. 13.874/19 (Art. 5), y la inserción de la metodología de análisis de impacto normativo (AIR) en el sistema sociojurídico. También apoya la propuesta el art. 174 de la Constitución Federal que, desde 1988, interfiere en el estado de los asuntos económicos para equilibrar las fuerzas del juego económico, por un lado, y para priorizar la empresa privada en la explotación de la actividad económica, por otro. Para ello, regula, supervisa, fomenta y planifica.

Sin embargo, para el nuevo estado nanotecnológico, es a partir de la reforma del la LINDB, que la ampliación de los espacios políticos de construcción normativa guiada por criterios de necesidad y eficiencia han ganado estructura, lenguaje propio y operatividad. Se ha posibilitado que los múltiples actores sectoriales tomen la iniciativa normativa en interés general de los implicados más allá de la actividad estatal, como se desprende de la lectura del artículo 29 de dicha Ley: en cualquier órgano, o poder de la edición de actos normativos por parte de la autoridad administrativa, salvo las que sean una mera organización interna, podrá ser precedida de una consulta pública a los interesados, preferentemente por medios electrónicos, que deberá ser considerada en la decisión. Además, pero no por ello menos importante, se prevé en el artículo 23 que, en caso de imposición de nuevos deberes o nuevas condiciones de derecho, el agente regulador deberá establecer un régimen de transición temporal para que el nuevo deber, o nuevo comportamiento cultural sea proporcional, equitativo, eficiente y sin perjuicio de los intereses generales.

Estas dos disposiciones previstas en la estructura del sistema se complementan con la reciente Ley de Libertad Económica, la cual, en su artículo $5^{\circ}$ dispone que la regulación por parte del Estado debe, desde entonces, preceder a la metodología de análisis de impacto normativo; en el cual reside la estructura del sandbox regulatorio y la propuesta de regulación del sistema nanotecnológico.

El sandbox, como se mencionó, está siendo utilizado en el momento de esta investigación por la Comisión de Bolsa y Valores (CVM) para regular el mercado de startups de FinTechs (Silva y Costa, 2019). Su objetivo es llevar este tipo de negocio tecnológico, propio de la Revolución Industrial 4.0, a la regulación legal para garantizar la sostenibilidad del negocio, la competencia leal con los bancos y la seguridad de los usuarios. Para poder construir el sandbox, la CVM está utilizando el análisis de impacto normativo a través de la consulta pública instituida por la Ordenanza CVM/PTE 48/2019.

El sandbox aplicado por la CVM es el resultado del establecimiento del "Laboratorio de Innovaciones Financieras" como un proyecto conjunto entre la Asociación Brasileña de Desarrollo, el Banco Interamericano de Desarrollo y la CVM, para, en un esfuerzo conjunto, desarrollar el sector financiero que opera a través de la innovación tecnológica. En este contexto, cabe advertir 
que Brasil “...ya posee el 32.7\% de toda la actividad de startups financieras en esta región del continente...” (Silva y Costa, 2019).

El referido decreto establece un procedimiento específico que precede a la regulación sectorial de las FinTechs por parte de la autoridad local en igualdad de condiciones con el mercado de capitales, que incluye la regulación específica y temporal de carácter experimental. En este marco temporal de regulación especial, la autarquía recoge datos empíricos para analizar y evaluar el impacto de cualquier regulación sectorial, esto es, los beneficios y procedimientos más adecuados para aplicar la solución recomendada.

Lo importante durante el proceso de sandbox es que las empresas implicadas en el experimento regulatorio - es decir, la industria - entreguen datos a la autarquía y así reduzcan la asimetría informativa tan perjudicial para la competencia y la regulación eficiente. Según Mello:

En el mundo de la informática, un sandbox es un entorno de pruebas cerrado diseñado para experimentar de forma segura con proyectos web o de software. Recientemente, y dentro de la acepción adoptada por el modelo de la CVM, el concepto se ha utilizado también en la economía digital para referirse a los entornos de prueba reglamentarios: cimientos de nuevos modelos de negocio que no están protegidos por la regulación actual ni supervisados por las instituciones reguladoras, pero que contienen en sí mismos cierto potencial de riesgo. (Mello, 2019).*

Según Silva y Costa, el sandbox regulatorio empleado por la CVM se utiliza desde hace tiempo en Reino Unido, Australia, Singapur y Hong Kong, y se caracteriza por "programas de duración limitada que permiten a las FinTechs, en fase inicial, probar sus ofertas en un entorno de mercado limitado, bajo la supervisión regulatoria de las agencias pertinentes, pero sin tener que obtener una licencia completa y someterse a las innumerables normas regulatorias de las innovaciones tecnológicas actuales" (Silva y Costa, 2019).

Con el fin de posibilitar el patrón metodológico de estandarización eficiente para todos los destinatarios y legitimados a la regulación, el gobierno federal, a través de la Casa Civil, elaboró la "Guía para la Elaboración de Análisis de Impacto Normativo" (Brasil, 2018) —el segundo párrafo único, del artículo $5^{\circ}$ de la Ley de Libertad Económica No. 13.874/19. El documento retrata el "diamante de la regulación" (Brasil, 2018), que significa elegir a través del marco, la mejor manera —además de la madurez del sistema socio-jurídico-, con la que el país aplicará el método a la intervención reguladora. Nótese que una de las modalidades de regulación ideal para cualquier sector es la corregulación, porque involucra la iniciativa pública y privada en la construcción de un patrón cultural de comportamiento económico que se asume como racional, virtuoso y proactivo.

\footnotetext{
* Traducción de los autores.
} 
Según la Guía, la regulación compartida a la corregulación:

[...] se produce cuando la industria desarrolla y administra sus normas, pero el gobierno proporciona apoyo legal para permitir su aplicación. En general, el gobierno establece normas o parámetros de calidad, o desempeño, permitiendo a los actores elegir la mayor forma de adecuar sus productos, procesos, servicios, y tecnologías, al desempeño esperado. (Brasil, 2018: 26). ${ }^{*}$

Por estas razones, lo ideal para el desarrollo de la industria 4.0, en donde se inserta la perspectiva disruptiva que trae la nanotecnología, será, a nuestro modo de ver, la aplicación del sandbox regulatorio experimental. Considerando que se presenta como una herramienta idónea para iniciar la regulación de la industria y, en consecuencia, orientar la toma de decisiones, generar bases de datos, y dirigir el control de los riesgos por parte de los múltiples actores e intereses involucrados durante el proceso reglamentario - a saber: laboratorios públicos y privados; empresas públicas y privadas; organizaciones representativas de los intereses ambientales y de los consumidores; agencias reguladoras complementarias; el propio gobierno a través del Ministerio de Ciencia, Tecnología, Comunicación e Innovación-MCTIC, por mencionar algunos.

En este sentido, estamos ante la Ley 4.0 para abordar las cuestiones tecnológicas $4.0 \mathrm{y}$, en consecuencia, sentar paulatinamente las bases de los avances en todas las áreas del derecho. El sandbox inaugura esta propuesta como una herramienta viable y hábil en la construcción de este derecho, completamente ausente en nanotecnologías. Y es que a decir de Feigelson:

[...] la regulación de las nuevas dinámicas puede darse en dos escenarios: (i) entorno de ausencia total de regulación. Es decir, espacios en los que no existen marcos legales ni estructuras instituidas para tratar los fenómenos en los que el principio de autonomía privada permite plenamente el desarrollo de la nueva dinámica (ej.: transporte privado a través de aplicaciones); y, (ii) entornos previamente regulados, pero no adecuadamente preparados para recibir las nuevas dinámicas creadas a partir del uso de las nuevas tecnologías (e.g.: FinTechs). (Feigelson, 2018).*

Feigelson explica que en Reino Unido, se ha aplicado el sandbox para volver a probar nuevos productos sin el riesgo de ser sancionado por el regulador que, a su vez, exige el cumplimiento de ciertas condiciones que se aplican adecuadamente al desarrollo nanotecnológico, a saber: i) consentimiento previo de los usuarios de nuevos productos; ii) límites financieros de las operaciones, e, iii) control de riesgos, como las dinámicas que detecten posibles fraudes (Feigelson, 2018). En Australia (ASICS, 2017), los requisitos son: i) innovación tecnológica en los productos; ii) demostración de benefi-

\footnotetext{
* Traducción de los autores.
} 
cios a los usuarios, e, iii) información sobre gestión de riesgos y protección del consumidor

Para Josanoff (2011), la frontera entre la ciencia y la política no está predeterminada, sino que se constituye a través de los propios procesos de asesoramiento de las instituciones políticas y de las pautas culturales que autorizan y, como resultado, condicionan la producción, recepción y asociación de conocimientos especializados para informar los procesos de regulación. Estos aspectos cumplen efectivamente el principio de precaución (Pellin y Engelmann, 2017) en la toma de decisiones sobre el desarrollo y el emprendimiento nanotecnológico y el tan ansiado control de riesgos para los sistemas sociales y ambientales de lo desconocido, desmedido y desinformado a la sociedad (según la escasez informativa al mercado). Por lo tanto, cumpliendo eficientemente con ahorro de tiempo, las inversiones y la seguridad precautoria, en la elaboración de normas legales se garantiza la continuidad del desarrollo, pero, desde entonces, sostenible democrático y legítimo, cuyo alcance deberá ser el cumplimiento de las agendas asumidas por la sociedad en cuanto a la información brasileña.

El papel principal podría ser asumido por la Agencia Nacional de Vigilancia Sanitaria, aunque la cartera pertenece al MCTIC, al ser un régimen especial (órgano independiente) y responsable del control de los productos y procesos destinados al consumo, cuya finalidad es precisamente la protección de la salud, tanto humana como de los ecosistemas, preocupándose por todo el ciclo de vida de un producto. El órgano MCTIC ya ha tenido la iniciativa infructuosa, debido a la absoluta falta de metodología e información sobre los datos generados por las nanotecnologías, así como el régimen jurídico inadecuado para el acceso a los datos almacenados y protegidos por el secreto industrial por los agentes de desarrollo, públicos y/o privados. Si el sandbox regulatorio de las nanotecnologías es conducido por la Agencia Nacional de Vigilancia Sanitaria, podría haber una garantía de eficiencia en los costos regulatorios, en la empresa nanotecnológica, y en la sociedad de manera rápida, democrática y efectiva.

\section{Conclusiones}

Dada la gran inversión en el desarrollo nanotecnológico y la aplicación de nanopartículas en productos y procesos destinados al consumidor, el riesgo ambiental y social está presente, debido a la falta de información técnico-científica sobre los posibles daños del uso de tal (nano)tecnología.

Teniendo en cuenta este contexto, la investigación tuvo como objetivo argumentar que el sandbox regulatorio puede servir como herramienta para construir un régimen legal que cubra los aspectos técnicos, científicos y legales, de acuerdo con las indicaciones sectoriales y los múltiples actores e intereses involucrados, de manera eficiente y experimental. También se advierte que este proceso de construcción puede ser facilitado por la Agencia Nacional 
de Vigilancia Sanitaria y deben establecerse ciertas condiciones como contrapartida a favor del ámbito regulatorio, como se ha hecho en otros países. Por lo tanto, es necesaria la adición de nuevas fuentes de Derecho 4.0, siendo su expresión inicial el sandbox regulatorio. El camino indicado para atender el proceso reglamentario de lo que no tiene normas regulatorias es analizar y evaluar que, a partir del debilitamiento del Estado, la corregulación entre los múltiples actores de normas jurídicas se ajuste a los altos estándares culturales sistémicos para el ejercicio de la actividad económica y la promoción del desarrollo económico según las directivas de la OCDE, así como de las derivadas del régimen jurídico-político establecido por la LINDB, la Ley de Libertad Económica, la Constitución Federal, los Derechos Humanos y la Agenda Global 2030.

La conclusión a la que se llega es que, si bien existe un pluralismo técnico-legal que gravita en torno a la gestión del tracto nanotecnológico que incluye inversión, desarrollo y riesgo, la construcción de una "Ciencia Reguladora" en el país está listada para ser elaborada a partir del sandbox, cuyo propósito epistemológico es equilibrar las fuerzas políticas del juego económico, las mejores técnicas y el régimen jurídico necesario para promover el desarrollo y controlar el riesgo en los sistemas sociales y medioambientales.

\section{Referencias}

Australian Securities and Investments Commision. (ASICS) "ASIC's regulatory sandbox." 21 de abril (2017). http://download.asic.gov.au/media/4221445/johnprice-speech-perth-fintech-meetup-published-21-april-2017.pdf(Consultado: 23 de septiembe, 2020).

Beck, Ulrich. (2011). Sociedade de risco: rumo a uma outra modernidade. 2a. ed. Traducción: Sebastião Nascimento. São Paulo: Editora 34.

Bobbio, Norberto. (2004). A era dos direitos. Traducción: Carlos Nelson Coutinho. Río de Janeiro: Elsevier.

Bobbio, Norberto. (2006). O positivismo jurídico: lições de filosofia do Direito. Traducción: Márcio Pugliesi, Edson Bini y Carlos E. Rodrigues. São Paulo: Ícone.

Brasil. (2013). Billing n ${ }^{\circ} 5.133$ de 2013. https://www.camara.leg.br/proposicoesWeb/ fichadetramitacao?idProposicao=600333 (Consultado: 3 de agosto, 2020).

Brasil. (2018). Diretrizes gerais e guia orientativo para elaboração de análise de impacto regulatório - AIR (PDF). Casa Civil. Edição: Casa Civil. 19 de octubre. http://www.casacivil.gov.br/central-de-conteudos/downloads/diretrizes-geraise-guia-orientativo_final_27-09-2018.pdf/@@download/file/Diretrizes\%20 Gerais\%20e\%20Guia\%20Orientativo_final_27.09.2018.pdf (Consultado: 23 de septiembre, 2019).

Brasil. (2010). Livro Azul: 4 Conferência Nacional de Ciência, Tecnologia e Inovação para o Desenvolvimento Sustentável. Centro de Estudos Estratégicos. http://www. cgee.org.br/documents/10195/734063/livroAzul_digital_18jan2011_6990. pdf/68c79d24-d589-42F5-ac66-4d6d728f9691?version=1.3 (Consultado: 19 de octubre, 2020). 
Brasil. (2002). Livro Branco: ciência, tecnologia e inovação. Ministério de Ciência, Tecnologia e Informação. http://seer.cgee.org.br/index.php/parcerias_estrategicas/article/viewFile/887/806 (Consultado: 7 de octubre, 2020).

Brasil. (2000). Sociedade da Informação no Brasil: Livro Verde. Edição: Organizado por Tadao Takahash. http://www.socinfo.org (Consultado: 20 de diciembre, 2020).

Brazilian Association of the Perfumery, Cosmetics and Personal Hygiene Industry (ABIHPEC). (2019). Annual ABIHPEC: Sector Panorama of 2019. ABIHPEC. Editado por ABIHPEC. http://abihpec.org.br/publicacao/panorama-do-setor-2019/ (Consultado: 7 de octubre, 2020).

Cai, Yuzhuo. (2013). Enhancing context sensitivity of the Triple Helix model: an institutional logics perspective. Triple Helix XI International Conference: The Triple Helix in a Context of Global change: continuing, mutating or unravelling? 8-10 de junio. http://www.triplehelixconference.org/th/11/bic/docs/Papers/Cai.pdf (Consultado: 15 de noviembre, 2020).

Engelmann, Wilson. (2018). Nanotecnologia e Direitos Humanos. Cadernos de Dereito Actual, 9: 441-487.

European Union (UE). (2009). A code of conduct for resonsible nanosciense and nanotechnology research. http://ec.europa.eu/research/sciense-society/document_ library/pdf_06/nanocode-apr09_en.pdf (Consultado: 7 de diciembre, 2020).

Feigelson, Bruno et al. (2019). Regulação 4.0. São Paulo: Thomson Reuters.

Feigelson, Bruno et al. (2018). sandbox: o futuro da regulação. 15 de enero. https://www. jota.info/opiniao-e-analise/colunas/regulacao-e-novas-tecnologias/sandbox-ofuturo-da-regulacao-15012018 (Consultado: 10 de agosto, 2020).

International Organization for Standardization (ISO). (2005). ISO International Organization for Standardization: when the world Agress. https://www.iso.org/ cmmittee/381983.html (Consultado: 10 de enero, 2021).

Jenik, Ivo, Lauer, Kate. (2017). Regulatory sandboxes and financial inclusion. CGAP Working Paper. Washington: CGAP, 1-22.

Josanoff, Sheila. (2011). The practices of objectivity in regulatory sciense. Social Knowledge in the Making, 307-337. https://press.uchicago.edu/ucp/books/book/ chicago/S/bo11753188.html (Consultado: 3 de octubre, 2021).

Kuhn, Thomas S. (2013). A estrutura das revoluções científicas. Traducción: Beatriz Vianna Boeira y Nelson Boeira. São Paulo: Perspectiva.

Lumann, Niklas. (2016). O direito da sociedade. Traducción: Saulo Krieger. São Paulo: Martins Fontes.

Mello, José Luiz Homem de. (2019). Nova regra da CVM lança as bases para adoção de modelo de sandbox Regulatório no Brasil. Cointimes. https://cointimes. com.br/ (Consultado: 18 de diciembre, 2020).

Miranda, Pontes de. (1972). Sistema de ciência positiva do direito: introdução à ciência do direito. 2o. vol. T. II. Río de Janeiro: Editor Borsoi.

Organization for Economic Cooperation and Development (OCDE). (2015). Policy environments and governance for innovation and sustainable growth through nanotechnology. 2 de febrero de 2015. http://www.oecd.org/officialdocuments/publicdisp laydocumentpdf/?cote=DSTI/STP/NANO(2013)13/FINAL\&doclangue =en 
(Consultado: 10 de octubre, 2020).

Ost, François. (1999). O tempo do direito. Traducción: Manuel Oliveira. Lisboa: Piaget.

Pellin, Daniela. (2019). A autorregulação regulada da Tríplece Hélice: a estruturação da boa governança em nanociência e nanotecnologia. Latvia: Nova Edições Acadêmicas.

Pellin, Daniela y Engelmann, Wilson. (2018). A política, a economia e o direito à efetividade do princípio da precaução: uma visão pluralista. En Aloísio Ruscheinsky, Cleide Calgaro e Thadeu Weller, Ética, direito socioambiental e democracia, 131146. Caxias do Sul: Educs.

Pellin, Daniela y Engelmann, Wilson. (2017). El principio legal de precaucion en scenario de riesgo nanotecnologico. Cadernos de Dereito Actual, 6: 09-29.

Pellin, Daniela y Engelmann, Wilson. (2019). O Brasil e a viamão do cumprimento da Agenda 2030: as empresas, as instituições e as nanotecnologias. Revista Culturas Jurídicas, 6 (jan-abr): 329-359.

Ruggiu, Daniele. (2011). Diritti humani e nanotecnologie in Europa: sul ruolo della Corte di Strasburgo. En Giorgia Guerra, Alessia Muratorio, Elena Pariotti, Mariassunta Piccini e Daniele Ruggiu, Forme di responsabilità, regolazione e nanotecnologie, 110-145. Bologna: Il Mulino.

Schwab, Klaus. (2016). A Quarta Revolução Industrial. Traducción: Daniel Moreira Miranda. São Paulo: Edipro.

Schwab, Klaus e Davis, Nicholas. (2018). Aplicando a Quarta Revolução Industrial. Tradução: Daniel Moreira Miranda. São Paulo: Edipro.

Silva, Luiza Caldeira Leite ye Costa, Gustavo. (2019). O sistema do sandbox regulatório como propulsor das novas tecnologias. 9 de enero. http://www.ab2I.org.br/osistema-de-sandbox-regulatorio-como-propulsor-de-novas-tecnologias-financeiras/ (Consultado: 18 de diciembre, 2020). 